\title{
ANXA11 regulates the tumorigenesis, lymph node metastasis and 5-fluorouracil sensitivity of murine hepatocarcinoma Hca-P cells by targeting $\mathrm{c}-\mathrm{Jun}$
}

\author{
Shuqing Liu ${ }^{1, *}$, Chunmei Guo ${ }^{2, *}$, Jiasheng Wang ${ }^{2, *}$, Bo Wang ${ }^{3}$, Houbao Qi ${ }^{2}$ Ming- \\ Zhong Sun ${ }^{2}$ \\ ${ }^{1}$ Department of Biochemistry, Dalian Medical University, Dalian 116044, China \\ ${ }^{2}$ Department of Biotechnology, Dalian Medical University, Dalian 116044, China \\ ${ }^{3}$ Department of Pathology, Dalian Medical University, Dalian 116044, China \\ *These authors have contributed equally to this work \\ Correspondence to: Ming-Zhong Sun, e-mail: smzlsq@163.com \\ Keywords: Anxal 1, hepatocarcinoma, lymphatic metastasis, chemoresistance, c-Jun \\ Received: October 20, $2015 \quad$ Accepted: February 09, $2016 \quad$ Published: February 18, 2016
}

\section{ABSTRACT}

Annexin A11 (Anxa11) is associated with various cancers. Using a pair of syngeneic murine hepatocarcinoma cells, Hca-P with 25\% and Hca-F with 75\% lymph node metastatic (LNM) potentials, we demonstrated Anxa11 involvement in hepatocarcinoma lymphatic metastasis. Here, ANXA11 acted as a suppressor for the tumorigenicity, LNM and 5-FU resistance of Hca-P via c-Jun. We constructed monoclonal Hca-P cell line with stable ANXA11 knockdown. Although Bax and Bcl2 levels increased in shRNA-Anxa11-transfected Hca-P, ANXA11 downregulation showed no clear effect on Hca-P apoptosis. ANXA11 downregulation promoted in vitro migration and invasion capacities of Hca-P. In situ adhesion potential of Hca-P cells toward LN was significantly enhanced following ANXA11 downregulation. Consistently, ANXA11 downregulation promoted the in vivo tumor growth and LNM capacities of Hca-P cells. ANXA11 knockdown enhanced the chemoresistance of Hca-P cells specifically toward 5-FU instead of cisplatin. Its downregulation increased c-Jun (pSer73) and decreased c-Jun (pSer243) levels in Hca-P. c-Jun (pSer243) downregulation seemed to be only correlated with ANXA11 knockdown without the connection to 5-FU treatment. Interestingly, compared with scramble-Hca-P cells, the levels of c-Jun and c-Jun (pSer73) in shRNA-Anxa11-Hca-P cells were upregulated in the presences of 0.1 and $1.0 \mathrm{mg} / \mathrm{L} \mathrm{5-FU}$. The levels changes from c-Jun and c-Jun (pSer73) in Hca-P cells showed a more obvious tendency with the combination of ANXA11 knockdown and 5-FU treatment. ANXA11 level regulates LNM and 5-FU resistance of Hca-P via C-Jun pathway. It might play an important role in hepatocarcinoma cell malignancy and be a therapeutic target for hepatocarcinoma.

\section{INTRODUCTION}

Hepatocellular carcinoma (HCC) is one of the most common cancers worldwide $[1,2]$. The lymphatic metastasis and chemoresistance are the major problems leading to its high recurrence with low post-surgical 5-years' survival [3-5]. Tumor metastasis is a multistep process, including the invasion of extracellular matrix (ECM), intravasation, translocation, migration and invasion of a secondary site, and finally the formation of metastatic nodules $[3,5]$. Deep study on the metastatic mechanisms can get the novel therapeutic targets and improve the prognosis for HCC.

Annexin A11 (Anxa11) is one member of annexins that are $\mathrm{Ca}^{2+}$-regulated phospholipid-binding proteins [6-10]. Like other annexins, the C-terminus of Anxa11 contains homologous tetrad annexin repeat core with $\mathrm{Ca}^{2+}$ binding sites. $\mathrm{Ca}^{2+}$ is competently and duly required for membrane binding, thermostability and tertiary structure of ANXA11. The N-terminus of Anxa11 is rich in glycine, tyrosine and proline residues with calcyclin (S100A6) and apoptosis-linked gene-2 protein (ALG-2) binding 
sites [11-13]. Anxa11 plays an important role in cell division, differentiation, apoptosis, vesicle trafficking and $\mathrm{Ca}^{2+}$ signaling. Anxa11 dysregulation is involved in the progression, drug-resistance, recurrence of systemic autoimmune disease and cancer [14-18].

c-Jun is a central component of transcription factor complex activator-protein 1 (AP-1) and a substrate of c-Jun-N-terminal kinases (JNKs) [19, 20]. c-Jun and its phosphorylation form p-c-Jun play important roles in cancer cell differentiation, invasion, metastasis, apoptosis, and chemoresistance through the action with other genes [21-24]. No study was reported on the association of c-Jun with the lymphatic metastasis or chemoresistance in HCC.

Hca-P with low lymph node metastatic (LNM) potential $(\sim 25 \%)$ and Hca-F with high LNM potential $(\sim 75 \%)$ were established in our research group as a pair of synogenetic murine HCC ascites cell lines with same genetic background [25-35]. They show the advantage of metastasizing specifically to LN without disseminating to other organs. They are the ideal experimental subjects for investigating the "pure" underlying LNM in the malignant hepatocellular tumors deriving from epithelia $[5,25-27$, $32,34-38]$. We previously reported the protein expression level of ANXA11 in Hca-P was 2-fold higher than Hca-F cells [39], suggesting ANXA11 might act as a potential suppressor for the lymphatic metastasis of murine HCC.

In this work, we investigated the effect of ANXA11 expression level on the malignant properties and underlying mechanism of Hca-P cells. Stable ANXA11 knockdown significantly enhanced the in vitro migration and invasion of Hca-P cells. ANXA11 downregulation also promoted the in vivo lymph node metastatic capacities of Hca-P cells. ANXA11 level regulated the lymphatic metastasis and 5-FU chemoresistance of Hca-P cells via c-Jun pathway.

\section{RESULTS}

\section{ANXA11 is stably downregulated in its monoclonal shRNA-transfected Hca-P cells}

Hca-P cells transfected with the specific shRNA of Anxall and with the shRNA of unrelated targeting sequence were named as shAnxa11-Hca-P and scrambleHca-P cells. The monoclonal shAnxa11-Hca-P and scramble-Hca-P cells were obtained by limited dilution against G418 screening. qRT-PCR and WB showed Axnall mRNA and ANXA11 protein levels were decreased by $82.49 \pm 3.49 \%(P<0.01$, Figure $1 \mathrm{~A})$ and $80.53 \pm 4.06 \%(P<0.01$, Figure 1B) in shAnxa11-Hca-P cells compared with scramble-Hca-P cells, while no difference was detected for its expression levels between scramble-Hca-P and Hca-P cells. The establishment of monoclonal shAnxa11-Hca-P cells with stable ANXA11 downregulation provided solid material for further study on the potential role of ANXA11 in murine HCC lymphatic metastasis.

\section{ANXA11 downregulation shows no clear effect on Hca-P cell apoptosis}

ANXA11 knockdown exhibits no effect on apoptosis of Hca-P cells. The influence of ANXA11 downregulation on Hca-P cell apoptosis was detected by flow cytometry and WB. Flow cytometry results (Figure 2A) showed there was no difference between the apoptosis rate of shAnxa11-Hca-P (5.87 $\pm 2.10 \%)$ cells and scramble-Hca-P $(4.24 \pm 2.25 \%)$ cells $(P>0.05)$. No statistical significant difference was determined for the expression levels of poly adeno ribose polymerase (PARP) and cleaved PARP in Hca-P cells following the knockdown of ANXA11 (Figure 2B). Although the levels of Bax and Bcl-2 were upregulated with statistical significances with $P<0.01$ and $P<0.05$ (Figure 2B) in shAnxa11-Hca-P compared with scramble-Hca-P cells, ANXA11 knockdown did not alter the expression level ratio of Bax/Bcl-2 $(P>0.05$, Figure 2B).

\section{ANXA11 knockdown promotes in vitro migration, invasion, in situ $\mathrm{LN}$ adhesion potential of Hca-P cells}

We reported ANXA11 linked to hepatocarcinoma lymphatic metastasis as its level was 2-fold higher in Hca-P than Hca-F cells [39]. The stable knockdown of ANXA11 on in vitro migration, invasion and in situ adhesion capacity to LN of Hca-P cells was performed. As shown in Figure 3, the numbers of migrated (106.0 \pm 29.7 , $P<0.01$; Figure $3 \mathrm{~A} 1$ and $3 \mathrm{~B})$ and invaded $(64.7 \pm 15.0$, $P<0.01$; Figure $3 \mathrm{~A} 2$ and $3 \mathrm{~B}$ ) shAnxa11-Hca-P cells increased to $253.6 \%$ and $217.8 \%$ of the numbers of $41.8 \pm 9.3$ and $29.7 \pm 8.4$ for scramble-Hca-P cells. ANXA11 knockdown also improved the in situ $\mathrm{LN}$ adhesion potential of Hca-P cells. shAnxa11-Hca-P cells showed a greater adhesive potential to inguinal and axillary LNs than scramble-Hca-P cells (Table 1). As the results shown in Figure 3C and 3D, the numbers of shAnxa11-Hca-P cells adhered to inguinal and axillary LNs were measured as $128.4 \pm 19.4$ and $98.8 \pm 10.1$ that were 2.1 - and 2.4-folds of $60.6 \pm 9.5$ and $42.0 \pm 6.0$ for scramble-Hca-P cells with statistical significances $(P<0.01)$. The above results indicate ANXA11 downregulation enhances the migration, invasion and adhesive capacities of Hca-P, which might result in its enhanced lymphatic metastasis malignancy.

\section{ANXA11 stable knockdown promotes in vivo tumorigenicity and LNM of Hca-P cells}

ANXA11 downregulation effect on tumorigenicity of Hca-P cells was investigated. shAnxa11-Hca-P and scramble-Hca-P cells were transplanted into the left footpads of mice. The sizes and volumes of the primary solid tumors formed on mice footpads were measured at $1,4,8,11,15,18$ and 21 days following 
cell inoculation. As the results showed in Figure 4A, ANXA11 knockdown exhibited promotion tendency to the volume of formed tumors (Figure 4A). The increase of tumor volumes showed statistical significances with $P<0.05$ in 11 and 15 days, and $P<0.01$ in 18 and 21 days for shAnxa11-Hca-P-transplanted mice compared with scramble-Hca-P-transplanted mice due to Anxa11 downregulation (Figure 4A). Figure 4B showed the images of the primary tumors after cell inoculations for 21 days, the tumor sizes of shAnxa11-Hca-Ptranspalnted mice were bigger than scramble-HcaP-transplanted ones. Consequently, there was a mass difference with stastical significance of the primary tumors formed on mouse footpad between the two group cells. After cell inoculations for 21 days, the masses for collected primary tumors from 10 shAnxa11-transplanted mice were $2.1330,1.6022,1.7563,1.7020,1.6747$, $0.3022,1.0448,1.2866,1.7573$ and $0.7332 \mathrm{~g}$ with an averaged mass of $1.3992 \pm 0.5566 \mathrm{~g}$. The masses of 9 (1 was lost when dissected it from footpad) scrambleHca-P-transplanted mice were measured as 0.7403, $1.2820,1.4073,1.0899,0.5000,0.0779,1.063,0.9171$ and $0.1423 \mathrm{~g}$ with an average of $0.8022 \pm 0.4764 \mathrm{~g}$. shAnxa11-transplanted mice showed $\sim 74.4 \%$ increased primary tumor mass than scramble-Hca-P-transplanted ones with stastical significance $(P=0.02309)$. ANXA11 downregulation inversely correlated in vivo tumor formation of Hca-P cells.
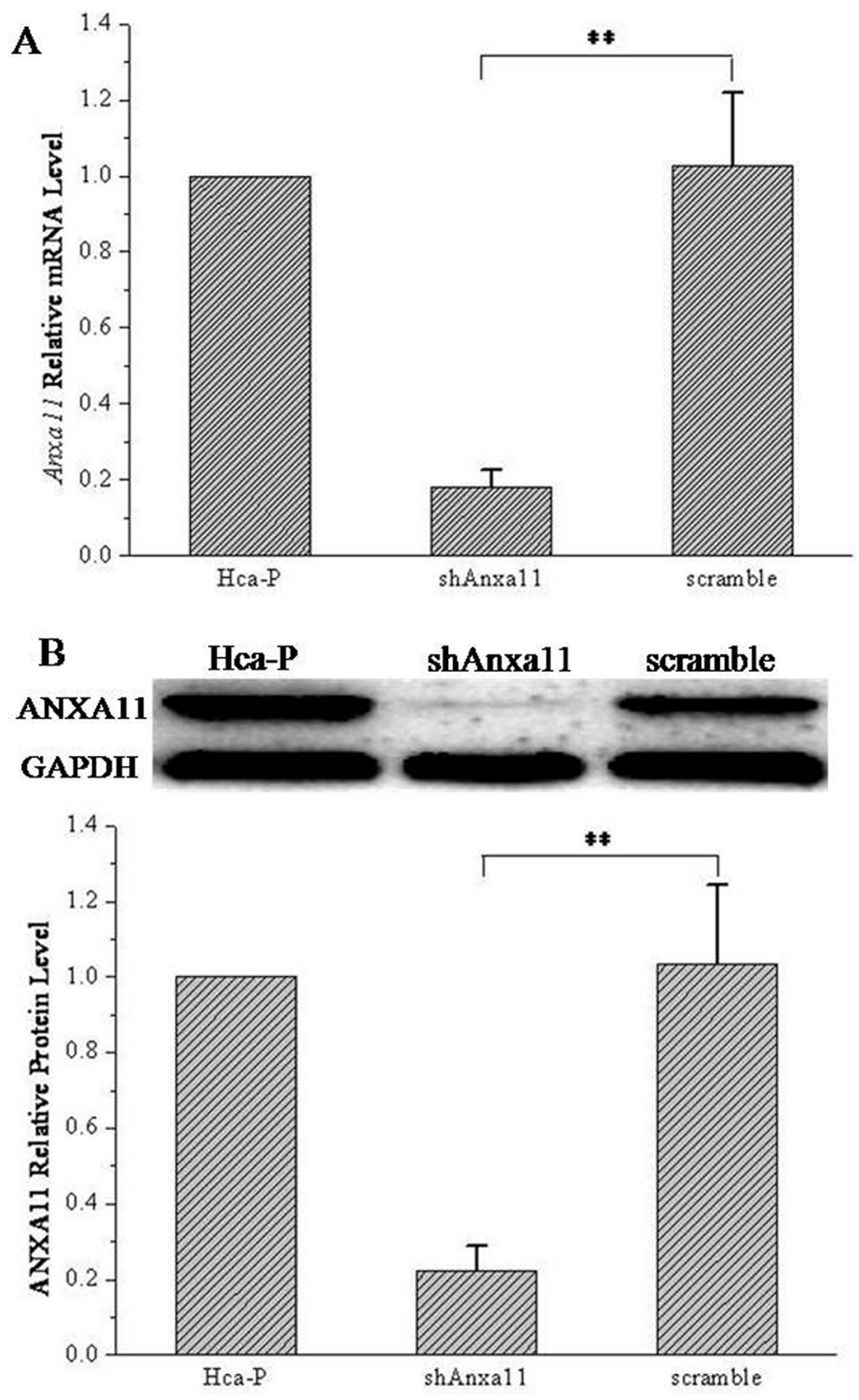

Figure 1: Anxa11 knockdown by RNAi. A. Relative Anxa11 mRNA levels in Hca-P, shAnxa11- Hca-P and scramble-Hca-P cells were determined by qRT-PCR using GAPDH as internal reference. B. WB assay of ANXA11 levels in Hca-P, shAnxa11-Hca-P and scrambleHca-P cells. GAPDH was the internal reference. Triplicate independent measurements were performed for WB assays. No statistical significances for the differences between Hca-P and scramble-Hca-P cells at both mRNA and protein levels for Anxa11. ${ }^{* *}$ Refers to the difference is of statistical significance $(P<0.01)$. 
Stable ANXA11 knockdown promoted the LNM of Hca-P cells. We found the number of LNs with invaded shAnxa11-Hca-P cells were more than that of LNs invaded with scramble-Hca-P cells. $2 / 6$ and $6 / 6$ collected inguinal and axillary LNs were with invaded shAnxa11Hca-P and scramble-Hca-P cells $(P<0.01)$. The structure of shAnxa11-Hca-P-metastasized LN was disorganized, while, in contrast, scramble-Hca-P-metastasized LN still retained in a relatively complete structure, as shown in Figure 4C. ANXA11 downregulation promoted the in vivo LNM capacity of Hca-P cells.

\section{ANXA11 knockdown enhances the chemoresistance of Hca-P cells to 5-FU}

5-FU and cisplatin are adjuvant drugs in the clinical treatment of $\mathrm{HCC}$ patients. CCK-8 assay was performed to investigate the influences of ANXA11 level on the drug sensitivity of Hca-P cells to 5-FU (Figure 5A) and cisplatin
(Figure 5B). No clear influence on Hca-P sensitivity to cisplatin administration (Figure 5B). In 5-FU concentration ranged in 0.01 to $10 \mathrm{mg} / \mathrm{L}$, the viabilities of shAnxa11Hca-P and scramble-Hca-P cells decreased dose-dependently (Figure 5A). shAnxa11-Hca-P showed a decreased sensitivity to 5-FU treatment than scramble-Hca-P cells (Figure 5A, $P<0.01$ ).

The influence of ANXA11 knockdown on the cell apoptosis induced by 5-FU was evaluated by Hoechst 33258 staining assay by determining the expression level change of cleaved PARP in Hca-P cells. Compared with the scramble-Hca-P cells, WB result indicated that the level of cleaved PARP induced by 5-FU administration was significantly reduced in shAnxa11-Hca-P cells following ANXA11 downregulation (Figure 5B). ANXA11 stable knockdown passivates the sensitivity of Hca-P cells to 5-FU-induced apoptosis (Figure 5C). Hoechst 33258 staining assay demonstrated that the condensed and fragmented nuclei of apoptotic cells were highly decreased

A
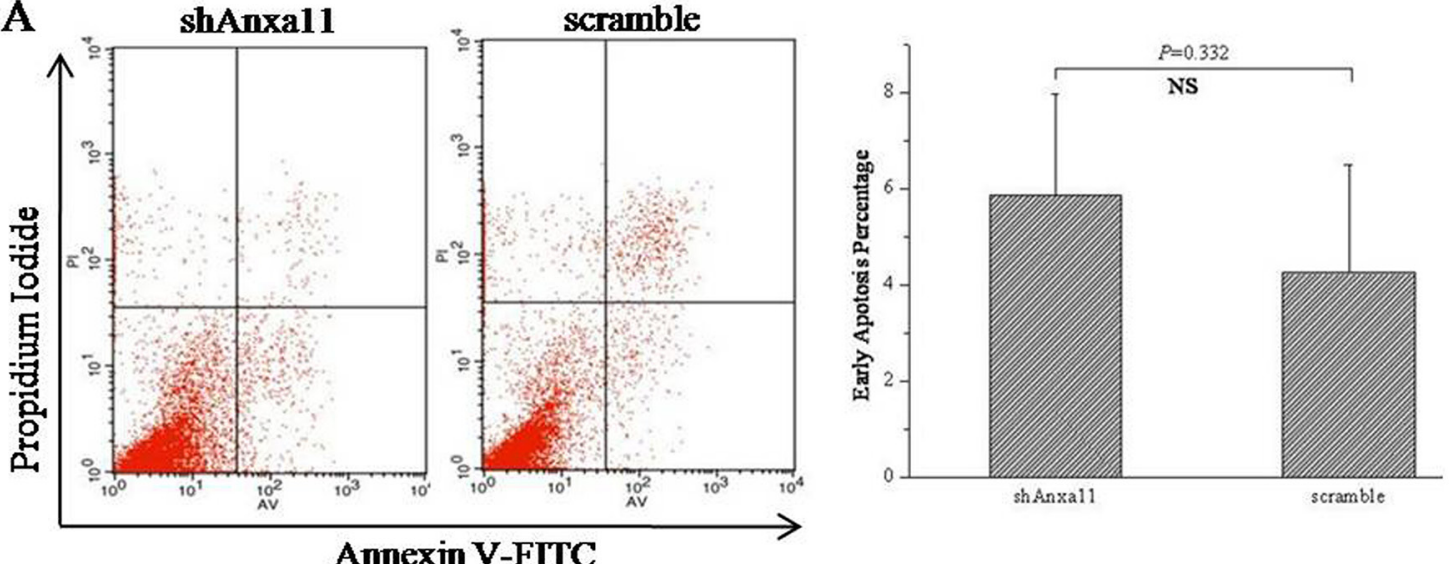

Annexin V-FITC
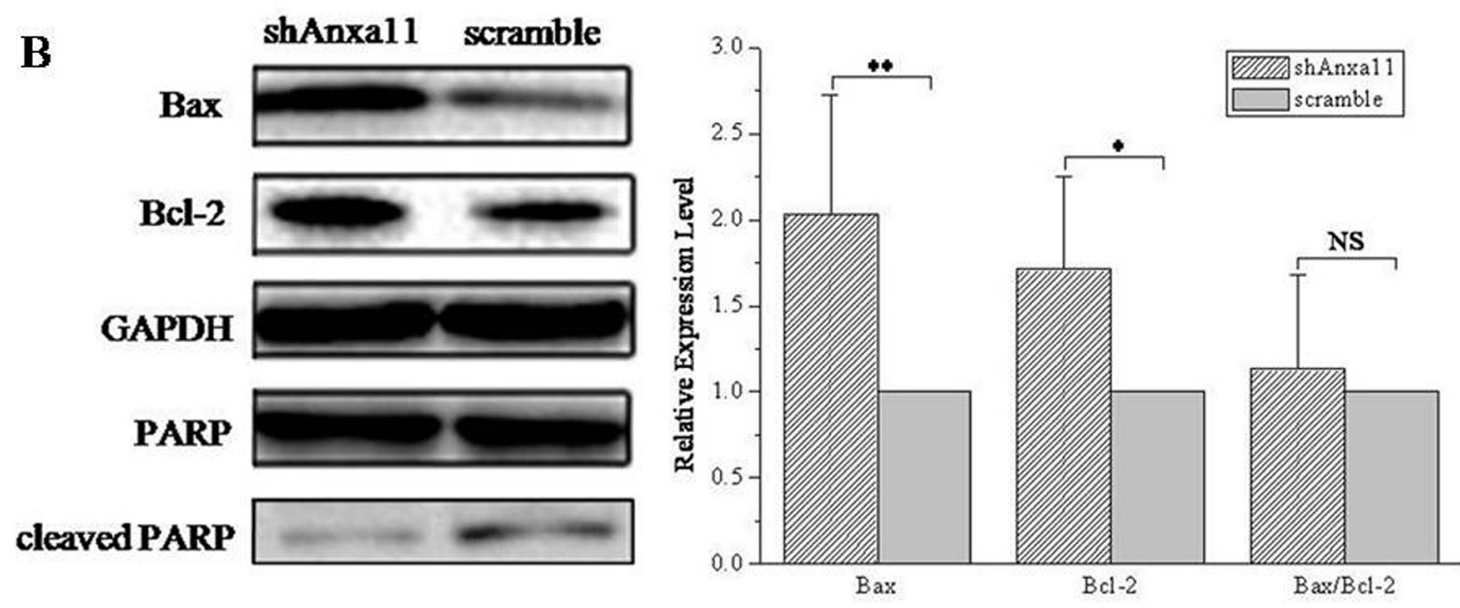

Figure 2: Anxa11 knockdown on Hca-P apoptosis. A. Flow cytometry showed Anxa11 level had no clear effect on Hca-P apoptosis $(P>0.05)$. B. WB assay of the levels of Bax, Bcl-2, PARP and cleaved PARP in shAnxa11-Hca-P and scramble-Hca-P cells. Anxa11 knockdown shows no clear effect on the levels of PARP and cleaved PARP. Bax $\left({ }^{* *} P<0.01\right)$ and Bcl-2 $\left({ }^{*} P<0.05\right)$ were up-regulated with statistical significances in shAnxa11-Hca-P compared with scramble-Hca-P cells. Anxa11downregulation exhibited no influence on Bax/ Bcl-2 ratio $(P>0.05)$. Three independent measurements were performed for each protein molecule. 
in shAnxa11-Hca-P cells in comparison with scrambleHca-P cells with the treatments of same concentrations of 5-FU (Figure 5C). ANXA11 downregulation enhances Hca-P cell chemoresistance to 5-FU specifically instead of cisplatin, which provides rational clinical treatment clue for HCC. It may be of potential application in chemotherapy of HCC patients.

\section{ANXA11 downregulation promotes Hca-P LNM and 5-FU resistance via c-Jun pathway}

The influence of ANXA11 knockdown on the expression levels of transcriptional factor c-Jun and its phosphorylated form was investigated using WB assay. Slight increase of c-Jun expression level was detected in shAnxa11-Hca-P cells to ANXA11 knockdown compared with scramble-Hca-P cells (Figure 6A), however, the difference was out of statistical significance (Figure 6B, $P>0.05$ ). The level of c-Jun (pSer73) was significantly increased in shAnxa11-Hca-P than scramble-Hca-P cells (Figure 6B, $P<0.01$ ). Interestingly, the level of c-Jun (pSer243) decreased (Figure 6A) in shAnxa11-Hca-P cells following ANXA11 downregulation (Figure 6B, $P<0.05$ ). These data suggest that Anxa11 regulates the malignant properties of Hca-P cells via c-Jun path.

ANXA11 is involved in the chemosensitivity of Hca-P to 5-FU via c-Jun path. Compared with scrambleHca-P cells, the expression levels of c-Jun increased in shAnxa11-Hca-P cells following 5-FU administrations with larger upregulation extent than c-Jun in Hca-P cells in responding to ANXA11 downregulation (Figure 6B). More importantly, the expression level difference of c-Jun between
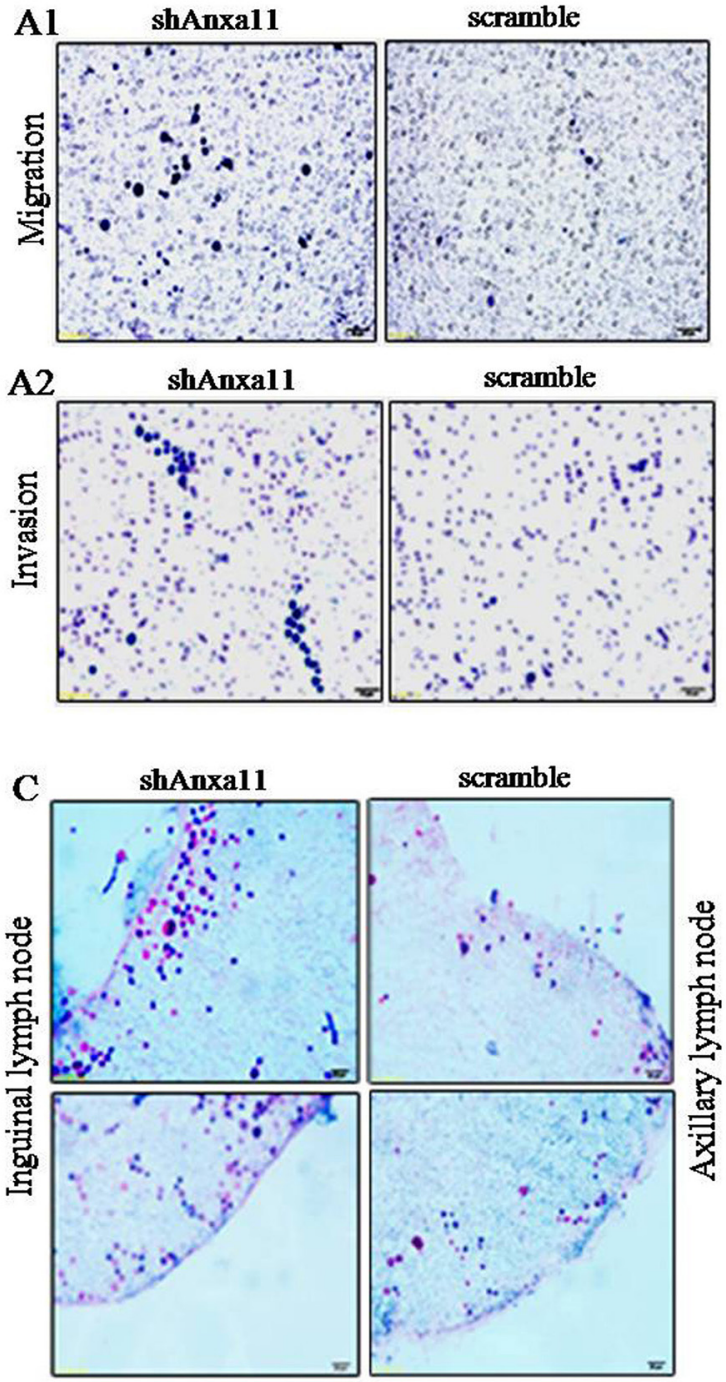

scramble

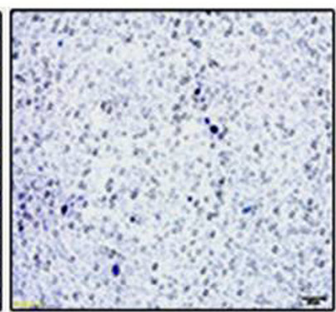

scramble

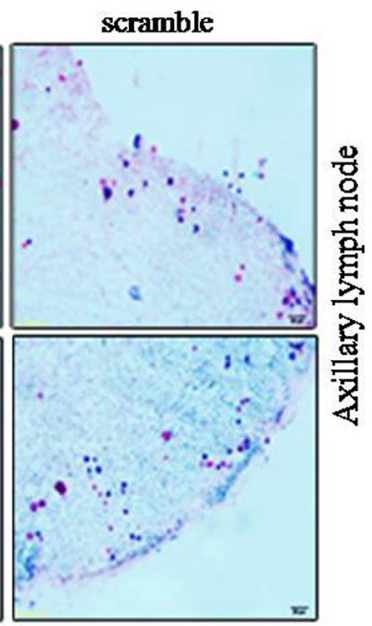

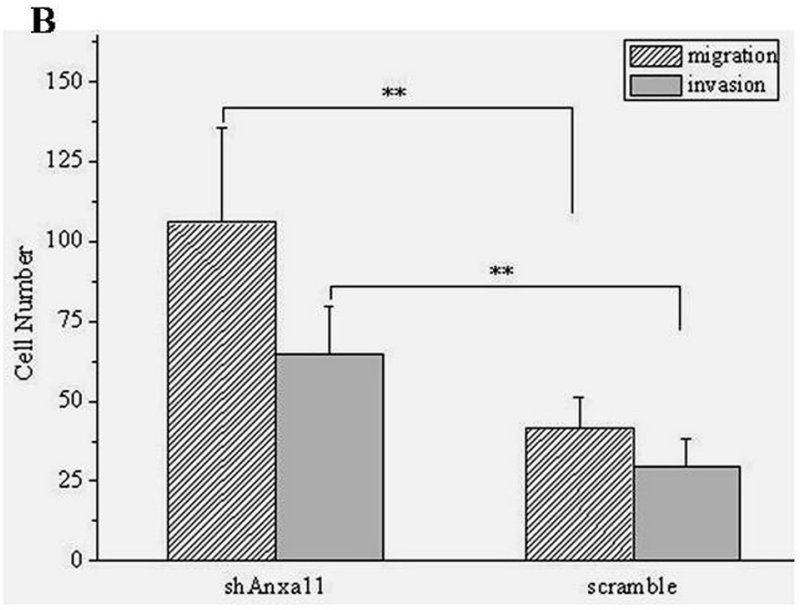

D

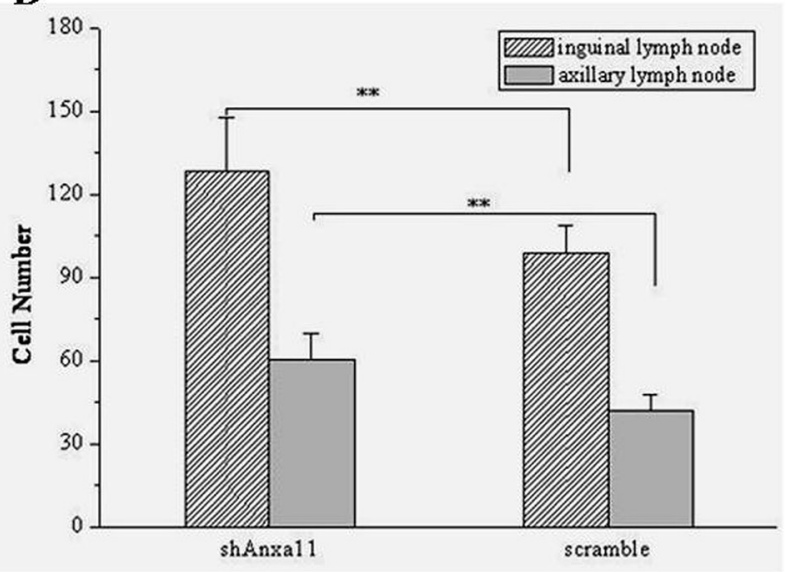

Figure 3: Influence of Anxa11 downregulation on in vitro migration, invasion and in situ LN adhesion potentials of Hca-P cells. A. and B. Anxa11 downregulation significantly enhanced the migration ability A1. and invasion capacity A2. of Hca-P cells, ${ }^{* *} P<0.01$. C. and D. Anxa11 downregulation significantly promoted in situ inguinal and axillary LNs adhesion capacities of Hca-P cells,

${ }^{* *} P<0.01$. Three independent measurements were performed for migration, invasion and LN adhesion assays. 
Table 1: ANXA11 knockdown on in situ adhesion ability of Hca-P cells to lymph node

\begin{tabular}{lccccccc}
\hline Cell & LN & \multicolumn{3}{c}{ Cell number } & \multicolumn{2}{c}{ Mean number } \\
\cline { 3 - 6 } & & Field 1 & Field 2 & Field 3 & Field 4 & Field 5 & $128.4 \pm 19.4$ \\
\hline shAnxa11-Hca-P & Inguinal & $138^{\text {a }}$ & 124 & 156 & 119 & 105 & $98.8 \pm 10.1$ \\
& Axillary & 113 & 91 & 96 & 105 & 89 & 68 \\
scramble-Hca-P & Inguinal & 70 & 46 & 58 & 61 & $60.6 \pm 9.5$ \\
& Axillary & 41 & 36 & 37 & 46 & 50 & $42.0 \pm 6.0$ \\
\hline
\end{tabular}

Note: ${ }^{a}$ Refers to the averaged number of adhered cells from three assays. Adhesion potential differences to inguinal and axillary LNs between shAnxa11-Hca-P and scramble-Hca-P cells were of statistical significance $(P<0.01)$.

shAnxa11-Hca-P and scramble-Hca-P cells (Figure 6A) at the concentrations of 0.1 and $1 \mathrm{mg} / \mathrm{L} \mathrm{5-FU}$ were of statistical significances $(P<0.01$, Figure 6B). The level of c-Jun (pSer243) was also detected decreased significantly (Figure $6 \mathrm{~A}$ and $6 \mathrm{~B}, P<0.05)$ in shAnxa11-Hca-P than scrambleHca-P cells, while, the downregulation extent was constant and comparable with its downregulation extent in scrambleHca-P following ANXA11 knockdown. Downregulation of c-Jun (pSer243) is induced by lower level of ANXA11 and not related to 5-FU treatment. c-Jun (pSer73) was found significantly upregulated in shAnxa11-Hca-P cells. Although comparable upregulation extents were determined for c-Jun (pSer73) levels in both shAnxa11-Hca-P and $0.01 \mathrm{mg} / \mathrm{L}$ 5-FU-treated-shAnxa11-Hca-P cells, its upregulation extents were calculated in shAnxa11-Hca-P with the treatments of 5-FU at 0.1 and $1.0 \mathrm{mg} / \mathrm{L}$ as $\sim 1.48$ - and 1.50 -folds of those in shAnxa11-Hca-P cells in comparisons with the corresponding controls (Figure 6B). ANXA11 knockdown and 5-FU administration seemed to show synergetic effect on c-Jun (pSer73) expression level (Figure 6B). It can be concluded ANXA11 downregulation interferes the properties of Hca-P through upregulating c-Jun (pSer73) and downregulating c-Jun (pSer243). These data suggest that Anxa11 is linked to lymphatic metastasis and 5-FU chemoresistance of Hca-P cells through c-Jun pathway.

\section{DISCUSSION}

As a member of annexin family, Anxa11 is essential to cell cycle, differentiation, apoptosis, vesicle trafficking and signal transduction $[6,7,40]$. It is linked to the autoimmune diseases and cancers [14, 16, 17, 41-43]. Anxa11 was involved in the LNM of colorectal cancer [14], the drug-resistant capacity of ovarian cancer cells or in the prognosis of ovarian cancer patients $[16,44]$. This study aimed to investigate the role of Anxa11 playing in LNM and drug resistance with the underlying action mechanisms for murine hepatocarcinoma cell. ANXA11 shows suppressor effect on the malignant behaviours of Hca-P cells. The stable knockdown of ANXA11 by RNAi (Figure 1) promoted the migration (Figure $3 \mathrm{~A} 1$ and $3 \mathrm{~B}$ ), invasion (Figure 3A2 and $3 \mathrm{~B}$ ) and $\mathrm{LN}$ adhesion capacities
(Figure 3C and 3D) of Hca-P cells in vitro as well as the tumorigenicity and LNM potentials (Figure 4) of Hca-P cells in vivo. In addition, ANXA11 knockdown increased the chemoresistance of Hca-P cells to 5-FU (Figure 5A) instead of cisplatin (Figure 5B). Current work indicates Anxa11 is closely associated with the LNM and 5-FU resistance of Hca-P cells, which suggests its potential application as an indicator for the lymphatic malignancy and chemoresistance of HCC.

Cancer cell survival is an important step of its metastasis [45]. The Bcl-2 families play important regulatory roles in cell apoptosis, acting either as the activator (Bax) or the inhibitor (Bcl-2). The balance between the levels of Bcl2 and Bax is critical for cancer cell survival. Increased Bax/ $\mathrm{Bcl}-2$ ratio contributes to cell apoptosis via caspase activation through mitochondrial pathway [46, 47]. Our work indicated that ANXA11 downregulation showed no affect on apoptosis of Hca-P cells without interrupting the Bax/Bcl-2 expression ratio and the expression levels of cleaved PARP (Figure 2). The deregulation of ANXA11 also showed on effects on the protein levels of caspase-3, caspase-8, caspase-9 and procaspase-8 (unshown data).

c-Jun pathway is linked to the growth, survival, metastasis, invasion and chemoresistance of a variety of cancer cells [21, 48, 49]. Phosphorylations of c-Jun at Ser73 [c-Jun (pSer73)] and Ser243 [c-Jun (pSer243)] are crucial for c-Jun transcriptional activity. Controversially, c-Jun (pSer73) increases, while, c-Jun (pSer243) inhibits [20] the transcriptional activity of c-Jun. c-Jun and JNK pathways were associated with the proliferation, migration and metastasis of HCC [5, 23, 24]. This work established the relationship between Anxa11 and c-Jun in lymphatic metastasis and 5-FU chemoresistance capacities of murine hepatocarcinoma Hca-P cells.

Although without affecting the cell apoptosis of Hca-P cells, ANXA11 stable knockdown (Figure 1) leads to enhanced in vitro migration and invasion capacities (Figure 3 ), in situ LN adhesion potential (Figure 3) and in vivo malignancy and LNM (Figure 4) of Hca-P cells. ANXA11 downregulation interferes the properties of Hca-P through upregulating c-Jun (pSer73) and downregulating c-Jun (pSer243). Following the downregulation of ANXA11, the 


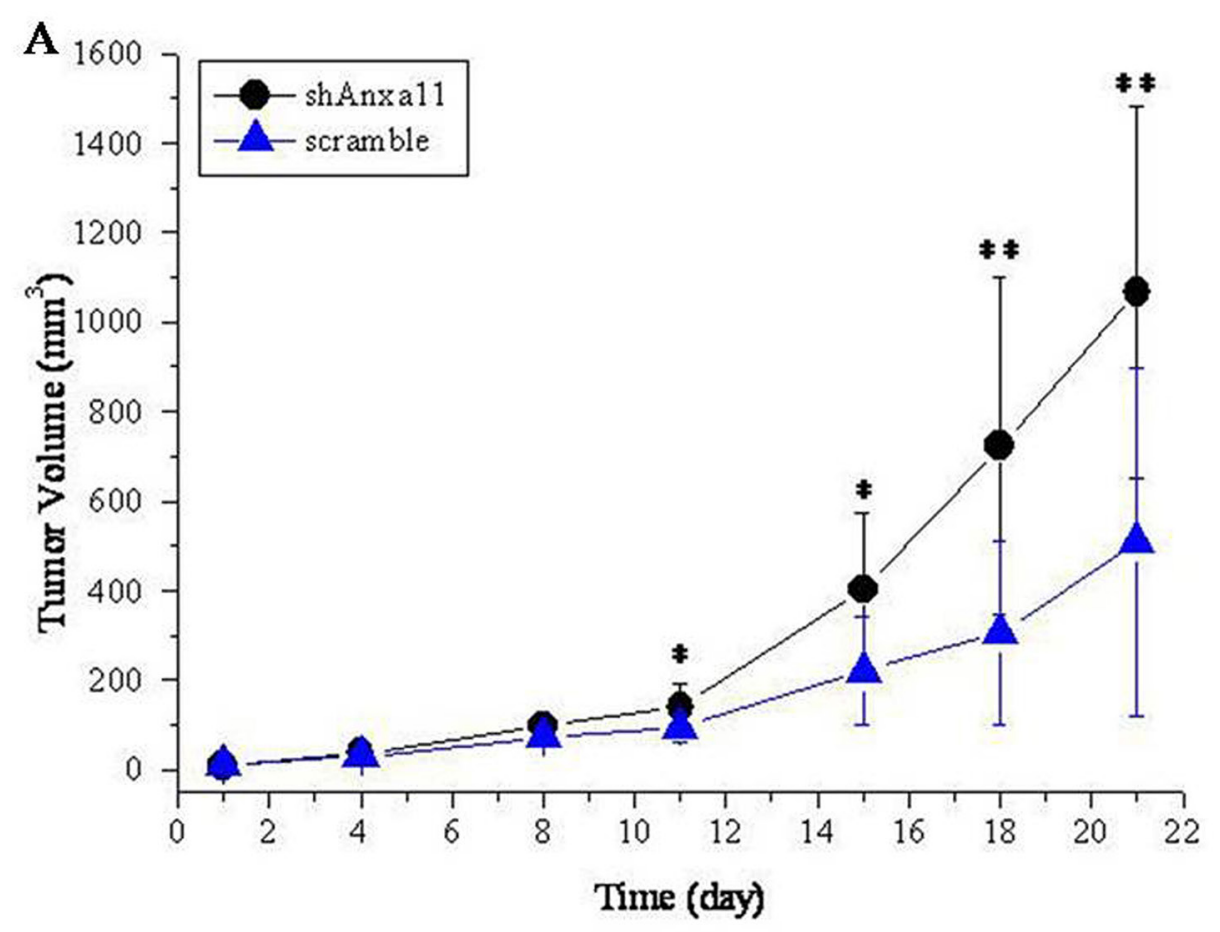

B
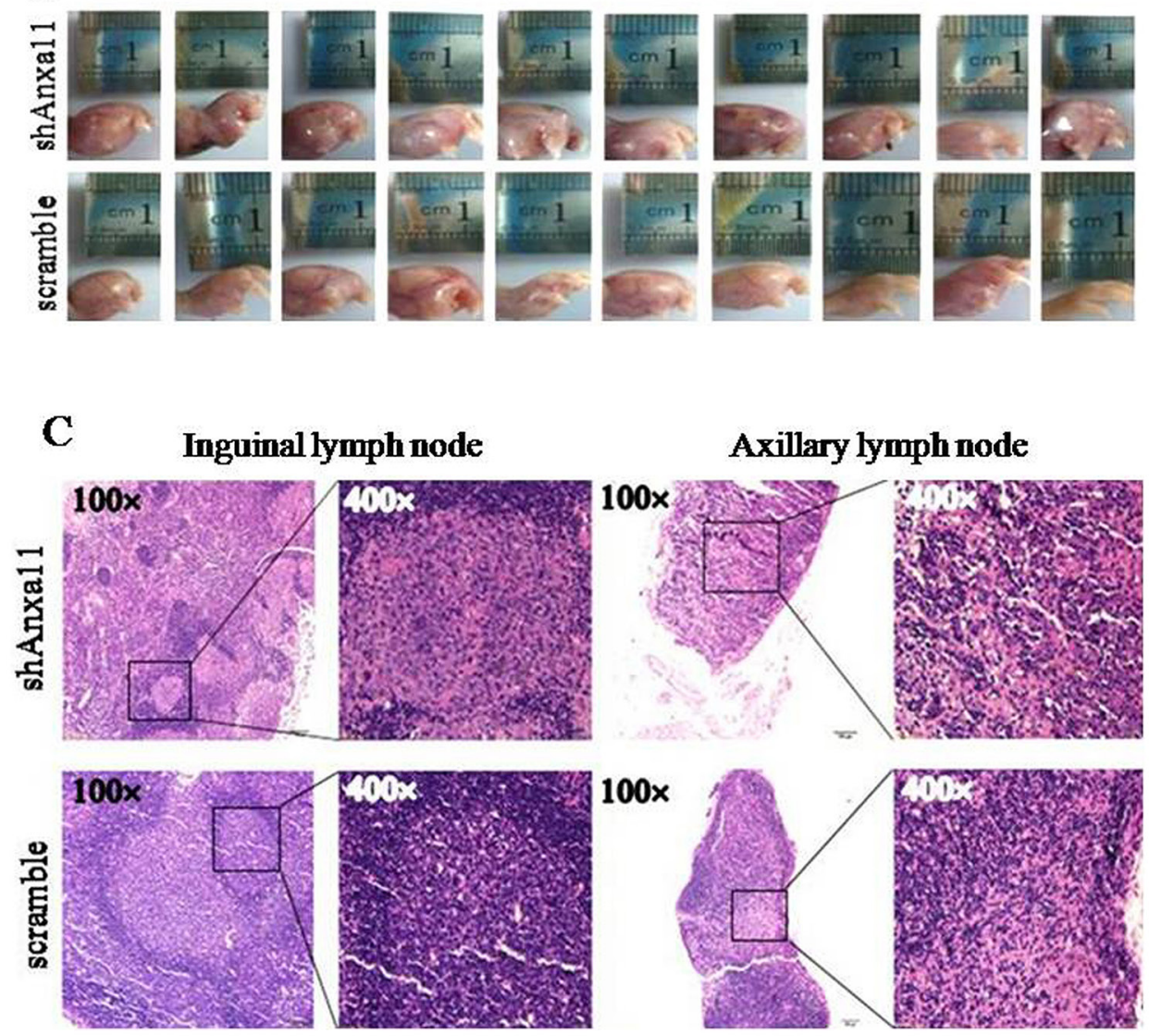

Figure 4: Anxa11 knockdown on tumorigenicity and LNM capacity for Hca-P cells. A. shAnxa11-Hca-P-transplaneted mice showed increased tumor growth speed than scramble-Hca-P-transplanted ones. Results were represented as the mean tumor volume \pm SD of each group. The tumor volume differences were with $P<0.05$ on the $11^{\text {th }}$ and $15^{\text {th }}$ days, and with $P<0.01$ on the $18^{\text {th }}$ and $21^{\text {st }}$ days after cell inoculation. B. Images of the primary tumors formed on the footpads of mice on the $21^{\text {st }}$ day after cell inoculations. C. Photos of inguinal and axillary LNs from shAnxa11-Hca-P-tanspanted mice and scramble-Hca-P-transplanted mice taken at the magnifications of $200 \times$ (left) and $400 \times$ (right). 

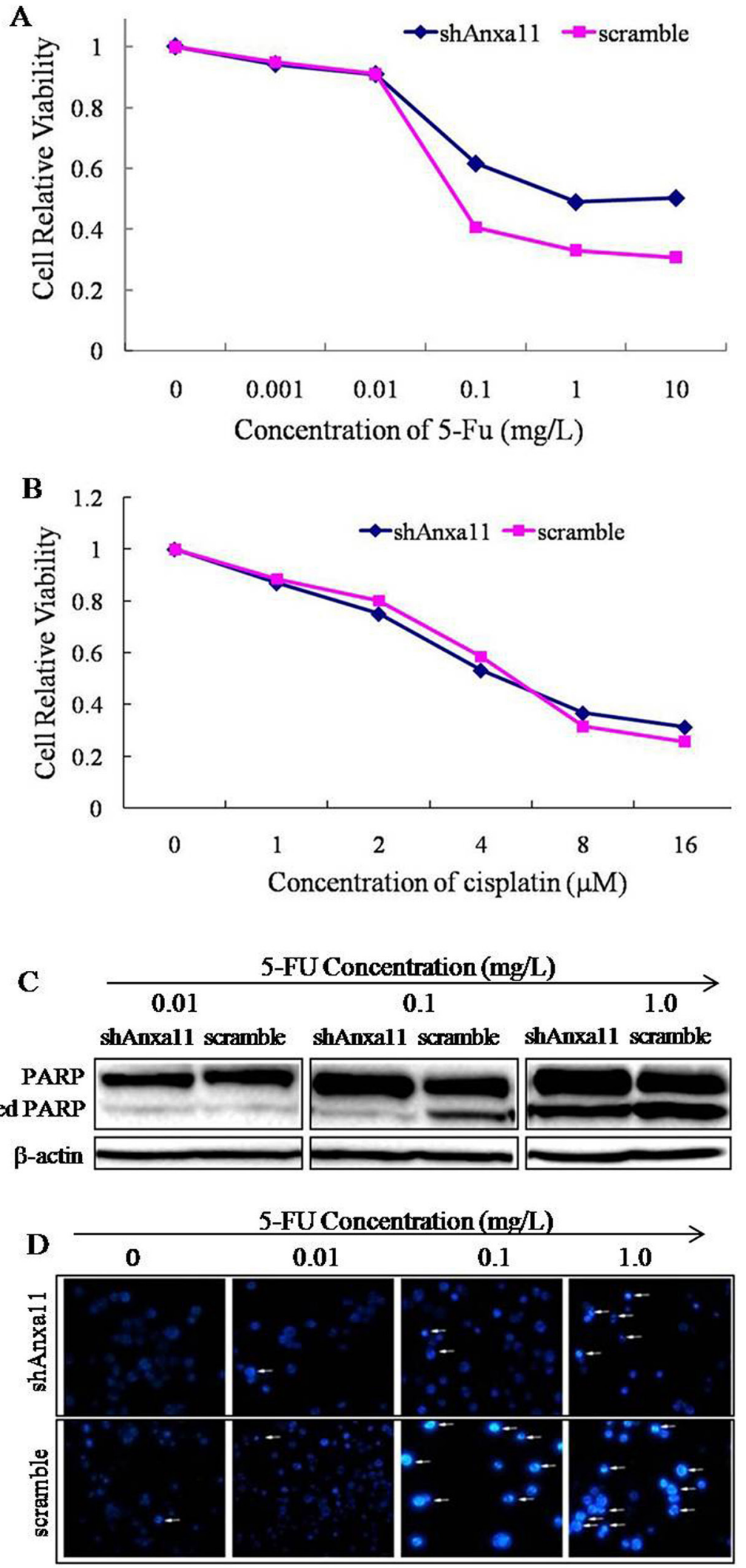

Figure 5: Anxa11 knockdown influence on Hca-P cell drug sensitivity to 5-FU and cisplatin. Dose-response curves for shAnxa11-Hca-P and scramble-Hca-P cells to 5-FU A. and cisplatin B. treatments. Cell viability was determined by CCK-8 assay. The viability differences between the two cell lines at $0.1,1.0$ and $10 \mathrm{mg} / \mathrm{L} 5$-FU administrations were of statistical significances $(P<0.01)$. C. Triplicate WB assays of the levels of cleaved PARP with the treatments of different concentrations of 5-FU in shAnxa11-Hca-P and scramble-Hca $-\mathrm{P}$ cells. D. Hoechst 33258 staining assay for shAnxa11-Hca-P and scramble-Hca-P cells with the treatments of different concentrations of 5-FU. 
expression level of c-Jun (pSer73) was significantly increased while the level of c-Jun (pSer243) was decreased in Hca-P cells (Figure 6). As the upregulation of c-Jun (pSer73) was predominantly evident than the downregulation of c-Jun (pSer243) (Figure 6), which might overally still activates the c-Jun signaling path to promote the in vitro and in vivo malignant behaviours of Hca-P cells.

ANXA11 knockdown regulates Hca-P chemoresistance to 5-FU via c-Jun pathway by up regulating the expression levels of c-Jun and c-Jun (pSer73). Following ANXA11 downregulation in Hca-P cells, the expression level of c-Jun increased in shAnxa11-Hca-P cells against 5-FU administrations at the concentrations of $0.1 \mathrm{mg} / \mathrm{L}$ and $1 \mathrm{mg} / \mathrm{L} 5$-FU with statistical significances $(P<0.01$, Figure $6 \mathrm{~B})$. The relative expression levels of c-Jun (pSer243) were consistently comparable in shAnxa11-Hca-P cells following ANXA11 knockdown and 5-FU treatments (Figure 6) suggesting Hca-P chemosensitivity to 5-FU is independent of c-Jun (pSer243). c-Jun (pSer73) upregulation extents calculated in shAnxa11-Hca-P with the treatments of 5-FU at 0.1 and $1.0 \mathrm{mg} / \mathrm{L}$ were $\sim 1.48$ - and 1.50 -folds of those in shAnxa11-Hca- P compared with the controls (Figure 6B).

A 5-FU Concentration (mg/L)
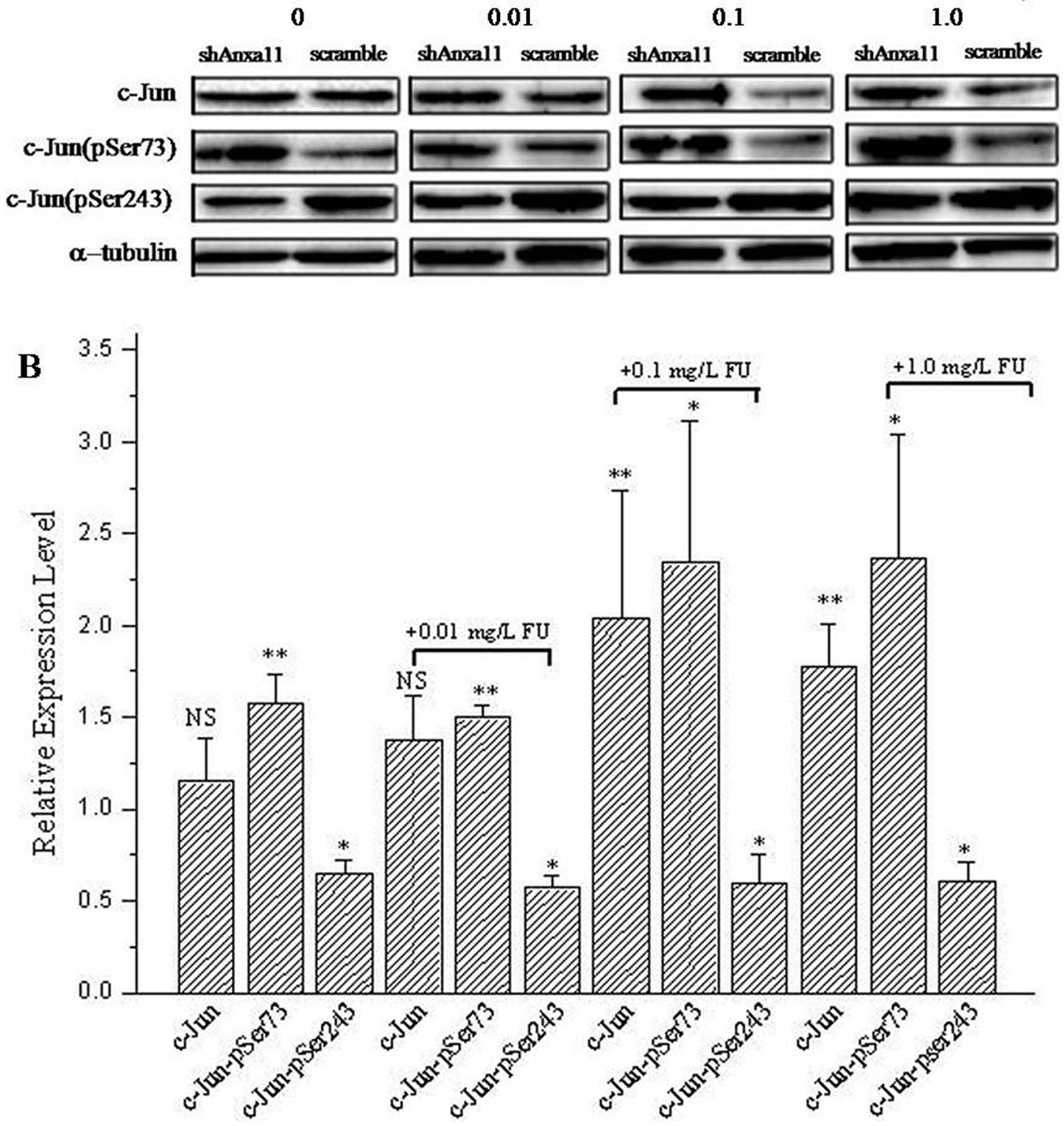

Figure 6: ANXA11 is linked to c-Jun pathway on Hca-P sensitivity to 5-FU. A. WB images of c-Jun, c-Jun (pSer73) and c-Jun (pSer243) in shAnxa11-Hca-P and scramble-Hca-P in 0, 0.01, 0.1 and $1.0 \mathrm{mg} / \mathrm{L}$ 5-FU. B. Quantified relative levels of c-Jun, c-Jun (pSer73) and c-Jun (pSer243) in shAnxa11-Hca-P vs scramble-Hca-P cells. Triplicate independent experiemnts were performed for each assay. ${ }^{*}$ and ${ }^{* *}$ refer to the differences are in statistical significances with $P<0.05$ and 0.01 , respectively. 
5-FU administration exhibits additive promotion on c-Jun (pSer73) expression level in Hca-P cells except for ANXA11 knockdown (Figure 6).

Taken together, current work shows ANXA11 exhibiting tumor suppressor effect on the in vitro and in vivo malignant behaviors of Hca-P cells. The stable knockdown of ANXA11 enhances the in vitro migration and invasion capacities, in situ LN adhesion potential, in vivo tumor malignancy and LNM, and chemoresistance to 5-FU of Hca-P cells. Anxa11 regulates the malignant properties and chemoresistance of Hca-P cells via c-Jun pathway. It is a potential indicator for the malignant progression and a specific therapeutical target of HCC.

\section{MATERIALS AND METHODS}

\section{Cell culture, animal experiment and ethics statement}

Murine hepatocarcinoma Hca-P cell line was maintained by our laboratory. Hca-P cells were grown in Chinese 615-mice (aged 6 weeks, weighting 20 \pm 2 g; Certificate of quality number: SCXK (Liao) 2008-0002) abdominal cavity for 7 days. Then the cells were incubated in 85\% RPMI 1640 (Gibco, USA) supplemented with 15\% fetal bovine serum (FBS: PAA, Australia) in a humidified environment at $37^{\circ} \mathrm{C}$ with $5 \% \mathrm{CO}_{2}$.

Mice were provided by the SPF Animal Laboratory Center of Dalian Medical University and treated and sacrificed following the protocols approved by the Experimental Animal Ethical Committee of Dalian Medical University (Permit Number: L2012012).

\section{Establishment of monoclonal ANXA11 knockdown Hca-P cell line}

Targeting shRNAs were designed according to Anxal1 mRNA sequence (Genbank: NM_013469.2) using siDirect and Whitehead. Anxa11-specific shRNA doublestranded oligodeoxyribonucleotides were designed as below: sense: 5'-GCGTGCCAAATCTGTATCCTTT-3' and antisense:5'-AAAGGATACAGATTTGGCACGC-3'. One shRNA with non-targeting sequence was designed as negative control as below: sense: 5'-GTTCTCCGAACGTGTCACGT-3' and antisense: 5'-ACGTGACACGTTCGGAGAAC-3'. The expression vectors of shRNA-Anxa11-pGPU6/GFP/Neo and shRNAscramble-pGPU6/GFP/Neo were constructed according to our previous publication $[29,35,39]$. Transfection was performed with Lipofectamine ${ }^{\mathrm{TM}} 2000$ (Invitrogen, USA) according to manufacturer's protocol. Stably transfected cells were screened against $400 \mu \mathrm{g} / \mathrm{mL}$ G418 for about 14 days, then the monoclonal shRNA-Anxa11-Hca-P (abbreviated as shAnxa11-Hca-P) and shRNA-scrambleHca-P (abbreviated as scramble-Hca-P) cells were obtained by limited dilution screening.

\section{Quantitative real-time PCR analysis}

Quantitative real-time PCR (qRT-PCR) was used to detect Anxa11 mRNA level in monoclonal shAnxa11Hca-P and scramble-Hca-P cell lines. In brief, total RNA was extracted from the cells using Trizol $^{\mathrm{TM}}$ reagent (Life technologies, USA). Reverse transcription was performed using PrimeScript ${ }^{\mathrm{TM}}$ RT reagent Kit with gDNA Eraser (TaKaRa, Japan). PCR was performed using an Agilent $\mathrm{M} \times 3005$ P real-time PCR machine (Agilent, USA) with FastStart universal SYBR Green Master (ROX) (Roche, Switzerland). PCR primers were listed below: GAPDH, F: 5'-GGTGAAGGTCGGTGTGAACG-3', R:5'-CTCGCTC CTGGAAGATGGTG-3'; Anxa11, F: 5'-GGTC CAACA AGCAGCGTC-3', R:5'-TCTCAAAGTTTCCCGACAG TTC-3'. The relative mRNA expression levels of Anxa11 among different group cells were analyzed using $2^{-\Delta \Delta C T}$ method [50].

\section{SDS-PAGE and western blotting (WB)}

Proteins were extracted using RIPA buffer [10 mM Tris- $\mathrm{HCl}$ (pH8.0), $150 \mathrm{mM} \mathrm{NaCl}, 1 \%$ Triton X-100, 0.5\% sodium deoxycholate, $0.1 \% \mathrm{SDS}]$ in the presences of 1 $\mathrm{mM} \mathrm{Na} \mathrm{VO}_{4}, 1 \mu \mathrm{g} / \mathrm{mL}$ leupeptin and $0.5 \mathrm{mM}$ PMSF. The supernatants were collected by centrifugation at 12000 rpm for $15 \mathrm{~min}$ at $4{ }^{\circ} \mathrm{C}$. Protein concentrations were determined by Bradford assay. Then, equal amounts of protein samples were boiled for $5 \mathrm{~min}$ in loading buffer, separated by $10 \%$ SDS-PAGE and transferred onto NC membrane (Millipore, MA). Being blocked in 5\% skim milk (in TBST) for $3 \mathrm{~h}$ at RT, the NC membrane was then incubated with primary antibodies shaking with $100 \mathrm{rpm}$ at $4{ }^{\circ} \mathrm{C}$ overnight. The primary antibodies were GAPDH (1:10000, KangChen, China), ANXA11 (1:1000, Proteintech, USA), Bax (1:1000, Cell Signaling, USA), Bcl-2 (1:500, Cell Signaling, USA), PARP (1:1000; Cell Signaling, USA), Pro-caspase 8 (1:1000, Cell Signaling, USA), c-Jun (1:1000, Sangon, China), phospho-c-Jun (1:1000, pSer73, Sangon, China), phospho-c-Jun (1:1000, pSer243, Sangon, China). The NC membranes were then washed with TBST $3 \times 10 \mathrm{~min}$, incubated with secondary antibody conjugated to horseradish peroxidase for $3 \mathrm{~h}$ at RT and washed again with TBST buffer $3 \times 10 \mathrm{~min}$. Protein bands were visualized by ECL (Advansta, USA) and detected using Bio-Rad ChemiDoc ${ }^{\mathrm{TM}}$ MP system (BioRad, USA).

\section{Cell apoptosis analysis by flow cytometry}

The effect of ANXA11 knockdown on Hca-P apoptosis was investigated using Annexin V-FITC/ propidium iodide apoptosis detection kit (KeyGEN, China) combined to flow cytometry assay. In brief, $1 \times 10^{6}$ cells were seeded into a $6-\mathrm{cm}$ dish, incubated at $37{ }^{\circ} \mathrm{C}$ with $5 \% \mathrm{CO}_{2}$ overnight and continuously cultured for $24 \mathrm{~h}$. The corresponding Hca-P cells from each group 
were harvested, washed with ice-cold PBS for 3 times, centrifuged at $1000 \mathrm{rpm}$ for $5 \mathrm{~min}$, resuspended in $500 \mu \mathrm{L}$ binding buffer, incubated in $5 \mu \mathrm{L}$ Annexin V-FITC and 5 $\mu \mathrm{L}$ PI in the dark for $30 \mathrm{~min}$ at RT. Finally, the cells were immediately subjected to FACSCalibur (BD Biosciences, USA). The results were analyzed using the CellQuest software (BD Biosciences, USA).

\section{In vitro cell migration and invasion assays}

Boyden transwell chamber assay was performed to detect ANXA11 downregulation on the migration and invasion capacities of Hca-P cells. For migration assay, $600 \mu \mathrm{L}$ of RPMI 1640 containing 15\% FBS were added into the lower chamber. Then, $5 \times 10^{4}$ cells from each group in $250 \mu \mathrm{L}$ serum-free RPMI 1640 were seeded into the upper chambers of transwell inserts with $8 \mu \mathrm{m}$ pore-size filters and incubated in a humidified incubator at $37{ }^{\circ} \mathrm{C}$ with $5 \% \mathrm{CO}_{2}$ for $24 \mathrm{~h}$. The non-migrated cells at the upper surface of filters were swabbed off using cotton swabs. The migrated cells at the lower chamber were fixed by $4 \%$ paraformaldehyde for $20 \mathrm{~min}$, stained by $0.1 \%$ crystal violet for $20 \mathrm{~min}$, washed with PBS, counted and averaged by selecting 5 random fields per well under a light microscope at a magnification of $100 \times$. Three independent experiments were performed for each assay.

For invasion assay, the $8 \mu \mathrm{m}$ I.D. polyester membrane fitters' surfaces of 24-well transwell units were coated with $50 \mu \mathrm{L}$ ice-cold ECM gel (1:8 dilution with RPMI 1640, Sigma-Aldrich, USA), incubated at $37^{\circ} \mathrm{C}$ for $3 \mathrm{~h}$ and then dried for $30 \mathrm{~min}$ at RT. $5 \times 10^{4}$ cells in $250 \mu \mathrm{L}$ serum-free RPMI 1640 were then loaded into the upper chamber. The rest procedures were the same as described in migration assay.

\section{In situ cell adhesion potential to lymph node (LN) assay}

In situ cell adhesion assay was performed to detect ANXA11 downregulation on Hca-P adhesion to LN. Briefly, fresh LNs taken from 615 mice were immediately frozen at $-20{ }^{\circ} \mathrm{C}$, buried by OCT and sectioned into 10 $\mu \mathrm{m}$ slices. The surfaces of frozen slices of inguinal and axillary LNs were covered in $200 \mu \mathrm{L}$ serum-free RPMI 1640 containing $1 \times 10^{5}$ cells from each group. The slices were then incubated at $37{ }^{\circ} \mathrm{C}$ with $5 \% \mathrm{CO}_{2}$ for 36 $\mathrm{h}$, washed with ice-cold PBS for 3 times, fixed in $95 \%$ ethanol for $5 \mathrm{~min}$, washed with flowing distilled water for $3 \mathrm{~s}$ and stained with hematoxylin eosin (HE). The number of adherent cells was counted using a microscope (Olympus, Japan) by randomly selecting five fields.

\section{5-FU and cisplatin sensitivity assay}

Cell counting Kit-8 (CCK-8) assay was performed to detect the influence of ANXA11 knockdown on the chemoresistances of Hca-P cells to 5-FU and cisplatin.
Cells from each group were seeded into a 96-well plate at a density of $7 \times 10^{3}$ cells/well in $100 \mu \mathrm{L}$ medium and cultured at $37^{\circ} \mathrm{C}$ with $5 \% \mathrm{CO}_{2}$ overnight. Then cells were treated with 5-FU with the final concentrations of $0,0.001$, $0.01,0.1,1,10 \mathrm{mg} / \mathrm{L}$, and with cisplatin with the final concentrations of $0,1,2,4,8$ and $16 \mu \mathrm{M}$, respectively. Being incubated at $37{ }^{\circ} \mathrm{C}$ with $5 \% \mathrm{CO}_{2}$ in 5-FUfor $48 \mathrm{~h}$ and cisplatin for $48 \mathrm{~h}$, the viability of the cells from each group was then measured using CCK- 8 assay. The absorbances at $450 \mathrm{~nm}$ were measured using a microplate reader (Thermo, USA). Cell viability ratio was defined as the absorbance of sample divided by the absorbance of control. Triplicate experiments were performed for each assay.

\section{Hoechst 33258 staining assay}

This assay was performed to investigate stable ANXA11 knockdown on Hca-P apoptosis to 5-FU induction. Cells from each group were treated with different concentrations of 5-FU for $48 \mathrm{~h}$, collected by centrifugation at $1000 \mathrm{rpm}$, washed with PBS buffer 3 times and fixed in $4 \%$ paraformaldehyde for $10 \mathrm{~min}$ at $4{ }^{\circ} \mathrm{C}$. Cells were then collected by centrifugation at $1000 \mathrm{rpm}$ for $5 \mathrm{~min}$, resuspended and washed with $2 \times$ $200 \mu \mathrm{L}$ PBS buffer. Then, the cells from each group were stained with Hoechst 33258 working solution in darkness for $10 \mathrm{~min}$ at $\mathrm{RT}$, washed with $\mathrm{ddH}_{2} \mathrm{O}$ for 3 times and immediately imaged using an inverted fluorescence microscope (Olympus, Japan) operated with the excitation wavelength of $340 \mathrm{~nm}$ at $200 \times$.

\section{In vivo tumorigenicity and LNM assay}

The effect of ANXA11 downregulation on the LNM of Hca-P cells was measured using Hca-P-transplanted mouse model. $1 \times 10^{6}$ cells in $30 \mu \mathrm{L}$ serum-free RPMI 1640 from each group were subcutaneously inoculated into the left footpads of inbred Chinese 615 mice. Each group had 10 mice with half male and half female. Following 1, 4, $8,11,15,18$ and 21 days after inoculation, the volumes of solid tumors formed in mice footpads were calculated according to the equation, $\mathrm{V}=\pi / 6 \times$ length $\times$ width $\times$ height, where the unit for length, width and height is in $\mathrm{mm}$. In 21 days after inoculation, the mice were sacrificed by collecting their LNs. For LNM analysis, the inguinal and axillary LNs were isolated and fixed in $10 \%$ neutral formalin, embedded in paraffin, sectioned into $4 \mu \mathrm{m}$ slices, stained by HE and examined by a light microscope (Olympus, Japan). LNM rates of each group cells were then obtained.

\section{Data processing and statistical analysis}

SPSS 17.0 software (SPSS Inc., Chicago, IL, USA) was used for all statistical analysis. All experimental date were represented as mean $\pm \mathrm{SD}$ of at least three independent experiments. The significant differences between the 
groups were analyzed using student's $t$ test. Statistical significant differences were labeled as ${ }^{*}$ and ${ }^{* *}$ for $P$ values below 0.05 and 0.01 .

\section{ACKNOWLEDGMENTS AND FUNDING}

This work was supported by grants from National Natural Science Foundation of China (No. 81050010, 81171957, 81272186 and 81100722), Outstanding Youth Scholar Growth Project in University and College of Liaoning (No. LJQ2011094) and Joint Fund of the Provincial Natural Science Foundation of Liaoning (No. 2014023047). The funders had no role in the study design, data collection and analysis, decision to publish, or preparation of the manuscript.

\section{CONFLICTS OF INTEREST}

The authors confirm that there are no conflicts of interest.

\section{Abbreviations}

ALG-2, apoptosis-linked gene-2 protein; Anxa11, Annexin A11; AP-1, activator-protein 1; Bax, Bcl-2 associated X protein; Bcl-2, B-cell lymphoma 2; CCK8 , cell counting kit-8; ECL, enhanced chemiluminescent; ECM, extracellular matrix; FBS, fetal bovine serum; FITC, fluorescein isothiocyanate; 5-FU, 5-fluorouracil; GAPDH, glyceraldehyde-3-phosphate dehydrogenase; Hca-F, hepatocarcinoma ascites cell F; Hca-P, hepatocarcinoma ascites cell P; HCC, hepatocellular carcinoma; HE, hematoxylin eosin; JNK, Jun-N-terminal kinase; LN, lymph node; LNM, LN metastasis; NC, nitrocellulose; OCT, optimum cutting temperature compound; PARP, poly adeno ribose polymerase; PBS, phosphate buffered saline; PI, propidium iodide; qRT-PCR, quantitative real-time polymerase chain reaction; RIPA, radioimmunoprecipitation assay; RNAi, RNA interference; RPMI, Roswell Park Memorial Institute; RT, room temperature; SD, standard deviation; SDS-PAGE, sodium dodecyl sulfate polyacrylamide gel electrophoresis; shRNA, short hairpin RNA; SPF, specific pathogen free; TBST, tris-buffered saline with Tween-20; WB, Western blotting.

\section{REFERENCES}

1. El-Serag HB. Hepatocellular carcinoma. N Engl J Med. 2011; 365:1118-1127.

2. Shen Q, Bae HJ, Eun JW, Kim HS, Park SJ. Shin WC, Lee EK, Park S. Park WS, Lee JY, Nam SW. MiR-101 functions as a tumor suppressor by directly targeting nemo-like kinase in liver cancer. Cancer Lett. 2014; 344:204-211.
3. Tang ZY. Hepatocellular carcinoma surgery-review of the past and prospects for the 21 st century. J Surg Oncol. 2005; 91:95-96.

4. Lyoo K, Hong SW, Kim SW, Yoon HY, Yoon SK. Silencing of 14-3-3zeta over-expression in hepatocellular carcinoma inhibits tumor growth and enhances chemosensitivity to cisdiammined dichloridoplatium. Cancer Lett. 2011; 303:99-107.

5. Hou Z, Ma W, Zhang J, Wang B, Huang Y, Zang S, Tang J, Li L. Enhanced tumorigenesis and lymphatic metastasis of CD133+hepatocarcinoma ascites syngeneic cell lines mediated by JNK signaling pathway in vitro and in vivo. Biomed Pharmacother. 2013; 67:337-345.

6. Tomas A, Futter C, Moss SE. Annexin 11 is required for midbody formation and completion of the terminal phase of cytokinesis. J Cell Biol. 2004; 165:813-822.

7. Williams LH, McClive PJ, Van Den Bergen JA, Sinclair AH. Annexin XI co-localises with calcyclin in proliferating cells of the embryonic mouse testis. Dev Dyn. 2005; 234:432-437.

8. Iino S, Sudo T, Niwa T, Fukasawa T, Hidaka H, Niki I. Annexin XI may be involved in $\mathrm{Ca}^{2+}$ - or GTP-gammaS -induced insulin secretion in the pancreatic $\beta$-cell. FEBS Lett. 2000; 479:46-50.

9. Gerke V, Creutz CE, Moss SE. Annexins: linking $\mathrm{Ca}^{2+}$ signalling to membrane dynamics. Nat Rev Mol Cell Biol. 2005; 6:449-461.

10. Wang J, Guo C, Liu S, Qi H, Yin Y, Liang R, Sun M-Z, Frederick G. Annexin A11 in disease. Clin Chim Acta. 2014; 431:164-168.

11. Moss SE, Morgan RO. The annexins. Genome Biol. 2004; $5: 219$.

12. Mizutani A, Watanabe N, Kitao T, Takumitsu H, Hidaka $\mathrm{H}$. The long amino-terminal tail domain of annexin XI is necessary for its nuclear localization. Arch Biochem Biophys. 1995; 318:157-165.

13. Barnes JA, Gomes AV. Proteolytic signals in the primary structure of annexins. Mol Cell Biochem. 2002; 231:1-7.

14. Duncan R, Carpenter B, Main LC, Telfer C, Murray GI. Characterisation and protein expression profiling of annexins in colorectal cancer. Br J Cancer. 2008; 98:426-433.

15. Kim JC, Ha YJ, Roh SA, Choi EY, Yoon YS, Kim KP, Hong YS, Kim TW, Cho DH, Kim SY, Kim YS. Feasibility of proposed single-nucleotide polymorphisms as predictive markers for targeted regimens in metastatic colorectal cancer. Br J Cancer. 2013; 108:1862-1869.

16. Song J, Shih Ie M, Chan DW, Zhang Z. Suppression of annexin A11 in ovarian cancer: implications in chemoresistance. Neoplasia. 2009; 11:605-614.

17. Hudelist G, Pacher-Zavisin M, Singer CF, Holper $T$, Kubista E, Schreiber M, Manavi M, Bilban M, Czerwenka $\mathrm{K}$. Use of high-throughput protein array for profiling of differentially expressed proteins in normal and malignant breast tissue. Breast Cancer Res Treat. 2004; 86:281-291.

18. Feng X, Zang S, Yang Y, Zhao S, Li Y, Gao X, Zhang J. Annexin A11 (ANXA11) gene polymorphisms are 
associated with sarcoidosis in a Han Chinese population: a case-control study. BMJ Open. 2014; 4:e004466.

19. Maeda S, Karin M. Oncogene at last-c-Jun promotes liver cancer in mice. Cancer Cell. 2003; 3:102-104.

20. Morton S, Davis RJ, McLaren A, Cohen P. A reinvestigation of the multisite phosphorylation of the transcription factor c-Jun. EMBO J. 2003; 22:3876-3886.

21. Shao J, Teng Y, Padia R, Hong S, Noh H, Xie X, Mumm JS, Dong Z, Ding HF, Cowell J, Kim J, Han J, Huang S. COP1 and GSK3- $\beta$ cooperate to promote c-Jun degradation and inhibit breast cancer cell tumorigenesis. Neoplasia. 2013; 15:1075-1085.

22. Hong IS, Kim SH, Koong MK, Jun JH, Lee YS, Kang KS. Roles of p38 and c-jun in the differentiation, proliferation and immortalization of normal human endometrial cells. Hum Reprod. 2004; 19:2192-2199.

23. Yang Z, Zhang Y, Wang L. A feedback inhibition between miRNA-127 and TGF- $\beta / c-J u n$ cascade in HCC cell migration via MMP13. PLoS One. 2013; 8:e65256.

24. Watanabe T, Hiasa Y, Tokumoto Y, Hirooka M, Abe M, Ikeda Y, Matsuura B, Chung RT, Onji M. Protein kinase $\mathrm{R}$ modulates c-Fos and c-Jun signaling to promote proliferation of hepatocellular carcinoma with hepatitis $\mathrm{C}$ virus infection. PLoS One. 2013; 8:e67750.

25. Cui X, Tang J, Hou L, Song B, Ban L. Identification of differentially expressed genes in mouse hepatocarcinoma ascites cell line with low potential of lymphogenous metastasis. World J Gastroenterol. 2006; 12:6893-6897.

26. Song B, Tang J, Wang B, Cui X, Hou L, Sun L, Mao L, Zhou C, Du Y, Wang L, Wang H, Zheng R. Identify lymphatic metastasis-associated genes in mouse hepatocarcinoma cell lines using gene chip. World J Gastroenterol. 2005; 11:1463-1472.

27. Hou L, Li Y, Jia Y, Wang B, Xin Y, Ling M, Lu S. Molecular mechanism about lymphogenous metastasis of hepatocarcinoma cells in mice. World J Gastroenterol. 2001; 7:532-536.

28. Li H, Ling M, Xie Y, Xie H. Establishment of a lymph node metastatic model of mouse hepatocellular carcinoma Hca-F cells in C3H/Hej mice. Oncol Res. 1998; 10:569-573.

29. Lin Q, Sun M-Z, Guo C, Shi J, Chen X, Liu S. CRKL overexpression suppresses in vitro proliferation, invasion and migration of murine hepatocarcinoma Hca-P cells. Biomed Pharmacother. 2015; 69:11-17.

30. Huang Y, Du Y, Zhang X, Bai L, Mibrahim M, Zhang J, Wei Y, Li C, Fan S, Wang H, Zhao Z, Tang J. Down-regulated expression of Annexin A7 induces apoptosis in mouse hepatocarcinoma cell line by the intrinsic mitochondrial pathway. Biomed Pharmacother. 2015; 70:146-150.

31. Zhang J, Song M, Wang J, Sun M-Z, Wang B, Li R, Huang Y, Hou L, Jin Y, Wang M, Tang J. Enoyl coenzyme A hydratase 1 is an important factor in the lymphatic metastasis of tumors. Biomed Pharmacother. 2011; 65:157-162.
32. Sun M-Z, Liu S, Tang J, Wang Z, Gong X, Sun C, Frederic G. Proteomics analysis of two mice hepatocarcinoma ascites syngeneic cell lines with high and low lymph node metastasis rates provide potential protein markers for tumor malignancy attributes to lymphatic metastasis. Proteomics. 2009; 9:3285-3302.

33. Du Y, Meng J, Huang Y, Wu J, Wang B, Ibrahim MM, Tang J. Guanine nucleotide-binding protein subunit beta2-like 1, a new Annexin A7 interacting protein. Biochem Biophys Res Commun. 2014; 445:58-63.

34. Song L, Mao J, Zhang J, Ibrahim MM, Li L, Tang J. Annexin A7 and its binding protein galectin-3 influence mouse hepatocellular carcinoma cell line in vitro. Biomed Pharmacother. 2014; 68:377-384.

35. Shi J, Meng L, Sun M-Z, Guo C, Sun X, Lin Q, Liu S. CRKL knockdown promotes in vitro proliferation, migration and invasion, in vivo tumor malignancy and lymph node metastasis of murine hepatocarcinoma Hca-P cells. Biomed Pharmacother. 2015; 71:84-90.

36. Wu J, Meng J, Du Y, Huang Y, Jin Y, Zhang J, Wang B, Zhang Y, Sun M-Z, Tang J. RACK1 promotes the proliferation, migration and invasion capacity of mouse hepatocellular carcinoma cell line in vitro probably by PI3K/Rac1 signaling pathway. Biomed Pharmacother. 2013; 67:313-319.

37. Huang Y, Wang Q, Du Y, Bai L, Jin F, Zhang J, Fan S, Wang H, Song L, Gao Y, Wang S, Tang J. Inhibition of annexin A7 gene and protein induces the apoptosis and decreases the invasion, migration of the hepatocarcinoma cell line. Biomed Pharmacother. 2014; 68:819-824.

38. Jia L, Wang S, Cao J, Zhou H, Wei W, Zhang J. siRNA targeted against matrix metalloproteinase 11 inhibits the metastatic capability of murine hepatocarcinoma cell Hca-F to lymph nodes. Int J Biochem Cell Biol. 2007; 39:2049-2062.

39. Liu S, Wang J, Guo C, Qi H, Sun M-Z. Annexin A11 knockdown inhibits in vitro proliferation and enhances survival of Hca-F cell via Akt2/FoxO1 pathway and MMP-9 expression. Biomed Pharmacother. 2015; 70:58-63

40. Tomas A, Moss SE. Calcium-and cell cycle-dependent association of annexin 11 with the nuclear envelope. J Biol Chem. 2003; 278:20210-20216.

41. van Venrooij WJ, Wodzig KW, Habets WJ, de Rooij DJ, van de Putte LB. Anti-56K: a novel, frequently occurring autoantibody specificity in connective tissue disease. Clin Exp Rheumatol. 1989; 7:277-282.

42. Jacobsen S, Halberg P, Ullman S, Khamashta MA, Asmussen K, Oxholm P, Jorgensen MK, van Venrooij WJ, Wiik A. Determination of autoantibodies to annexin XI in systemic autoimmune diseases. Lupus. 2000; 9:515-520.

43. Misaki Y, Van Venrooij WJ, Pruijn GJ. Prevalence and characteristics of anti-56K/annexin XI autoantibodies in systemic autoimmune diseases. J Rheumatol. 1995; 22:97-102. 
44. Song J, Shih Ie M, Salani R, Chan DW, Zhang Z. Annexin $\mathrm{XI}$ is associated with cisplatin resistance and related to tumor recurrence in ovarian cancer patients. Clin Cancer Res. 2007; 13:6842-6849.

45. Soldani C, Scovassi AI. Poly(ADP-ribose) polymerase-1 cleavage during apoptosis: an update. Apoptosis. 2002; 7:321-328.

46. Sadidi M, Lentz SI, Feldman EL. Hydrogen peroxideinduced Akt phosphorylation regulates Bax activation. Biochimie. 2009; 91:577-585.

47. Xin M, Deng X. Nicotine inactivation of the proapoptotic function of Bax through phosphorylation. J Biol Chem. $2005 ; 280: 10781-10789$.
48. Chen L, Qian L, Song Y, Yuan G, Zhang H, Yu M, Hu M, Shen B, Guo N. c-Jun, a crucial molecule in metastasis of breast cancer and potential target for biotherapy. Oncol Rep. 2007; 18:1207-1212.

49. Sze KM, Chu GK, Lee JM, Ng RO. C-terminal truncated hepatitis $\mathrm{B}$ virus $\mathrm{x}$ protein is associated with metastasis and enhances invasiveness by c-Jun/matrix metalloproteinase protein 10 activation in hepatocellular carcinoma. Hepatology. 2013; 57:131-139.

50. Livak KJ, Schmittgen TD. Analysis of relative gene expression data using real-time quantitative PCR and the 2(-Delta Delta C(T)) Method. Methods. 2001; 25:402-408. 